\title{
Interfaces
}

INTERFACES Image Texte Language

$44 \mid 2020$

Les manières de faire vernaculaires

\section{Du vernaculaire comme genre cinématographique}

\section{Gala Hernández López}

\section{(2) OpenEdition}

\section{Journals}

Édition électronique

URL : http://journals.openedition.org/interfaces/1767

DOI : 10.4000/interfaces. 1767

ISSN : 2647-6754

Éditeur :

Université de Bourgogne, Université de Paris, College of the Holy Cross

\section{Édition imprimée}

Date de publication : 15 décembre 2020

Pagination : 125-144

ISSN : 1164-6225

\section{Référence électronique}

Gala Hernández López, « Du vernaculaire comme genre cinématographique », Interfaces [En ligne], 44 | 2020, mis en ligne le 15 décembre 2020, consulté le 21 décembre 2020. URL : http:// journals.openedition.org/interfaces/1767 ; DOI : https://doi.org/10.4000/interfaces.1767

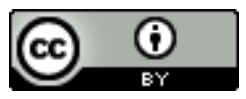

Les contenus de la revue Interfaces sont mis à disposition selon les termes de la Licence Creative Commons Attribution 4.0 International. 


\title{
DU VERNACULAIRE COMME GENRE CINÉMATOGRAPHIQUE
}

\author{
Gala Hernández López \\ Université Paris 8 Vincennes Saint-Denis, ESTCA
}

\begin{abstract}
Résumé : Le présent article propose une (re)définition du qualificatif «vernaculaire» appliqué à la vidéo. En premier lieu, nous dessinons une brève généalogie de l'utilisation de l'expression «vidéo vernaculaire» dans la littérature scientifique anglophone et francophone. Ensuite, nous tentons d'énumérer les caractéristiques essentielles de la vidéo vernaculaire tout en constatant la «vernacularisation» subie par le media vidéographique dans la première décennie de notre siècle. Par la suite, nous lançons l'hypothèse d'un progressif processus d'institutionnalisation ou canonisation de la vidéo vernaculaire, qui aurait eu lieu depuis les années 2010. Finalement, nous exposons trois exemples de réemploi cinématographique de vidéos vernaculaires qui montrent la réappropriation du vernaculaire par l'autorité artistique et nous en révélons les enjeux politiques et esth-éthiques.
\end{abstract}

Mots-clefs : vidéo vernaculaire, YouTube, web participatif, réseaux sociaux, médias sociaux, Contenus Générés par les Utilisateurs, vernacularisation, appropriation

Abstract: This article proposes a (re)definition of the qualifier "vernacular" applied to video. First, we draw a brief genealogy of the use of the term "vernacular video" in English and French academic literature. Next, we try to list the essential characteristics of the vernacular video while noting the "vernacularization" undergone by the videographic media in the first decade of our century. Then, we hypothesize a progressive process of artistic institutionalization of the vernacular video which may have taken place since the 2010s. Finally, we present three examples of cinematographic appropriation of vernacular videos, showing the appropriation of vernacular videos by the artistic authority, and we reveal the political and aesth-ethical stakes of these practises.

Keywords: vernacular video, YouTube, participative web, social networks, social media, User-Generated Content, vernacularisation, appropriation 
Le 6 octobre 1960, le cinéaste expérimental Jonas Mekas écrivait ces mots dans son journal:

Les films seront bientôt aussi faciles à réaliser que les poèmes écrits et presque aussi bon marché. Ils seront réalisés partout et par tout le monde. Les empires du professionnalisme et des gros budgets s'effritent. [...] C'est la meilleure chose qui soit arrivée au cinéma depuis que Griffith tourna son premier gros plan. (Mekas 26)

Ces mots prophétiques de Mekas s’inscrivent sous le titre «On film troubadours», « À propos des troubadours cinématographiques». Les troubadours étaient ceux qui savaient trouver « les bons mots et les belles musiques» («bos motz e gai sons») (Marrou 79). L'étymologie occitane du mot, trobador, veut dire précisément trouveur. La référence du cinéaste lituanien est spécialement pertinente car, à partir de la seconde moitié du XII ${ }^{\mathrm{e}}$ siècle, les œuvres littéraires, circulant oralement, étaient en général anonymes et, lorsqu'il y avait textes et qu'ils étaient signés, on ne savait pas si c'était «par l'auteur, le récitant ou encore le copiste» (Neeman 10). Il s'agit-là d'une comparaison fort heureuse de la part de Mekas, qui ne pouvait savoir en 1960 que le coût de production des images en mouvement diminuerait drastiquement avec le numérique, transformant tout un chacun en filmeur du réel, mais aussi que les futurs troubadours cinématographiques seraient des trouveurs numériques, ces copistes anonymes que nous sommes tous devenus, désormais spectateurs et passeurs, jongleurs d'un mondeimage fragmenté sur nos écrans. En effet, à l'ère post-internet ${ }^{1}, 1^{\prime}$ 'auctorialité est, tout comme du XII ${ }^{\mathrm{e}}$ au XIV ${ }^{\mathrm{e}}$ siècle, «plurielle, collective» (Neeman 10). L'originalité ou l'authenticité des contenus ne sont que des critères dilués parmi d'autres pour en évaluer l'intérêt. La propriété intellectuelle est, dans les deux époques, remise en question, ou même directement ignorée. On en trouvera une illustration frappante dans le phénomène des mèmes, par lequel des images, dont l'identité de l'auteur est inconnue, ou du moins clairement accessoire, sont reprises et déclinées en masse sur l'internet: ce qui compte, ce n'est pas tant d'avoir créé, produit l'image, mais de l'avoir trouvée et de la faire circuler. Si, du fait de l'emprise de la tradition, «la réécriture (remaniement, combinaison de plusieurs textes ou encore continuation d'un texte antérieur) est le fondement de la création littéraire au Moyen Âge» (Neeman

Nous utilisons le terme «post-internet» d'après la définition de l'artiste-chercheur Grégory Chatonsky, pour qui le préfixe «post» n'a pas une connotation chronologique, mais plutôt une dimension réflexive et critique, de la même manière que la postmodernité signifiait une réaction à l'échec du projet de modernité. Ce n'est pas que nous vivons «après » internet, bien au contraire. C'est que nous vivons tellement dans l'internet, c'est que les réseaux déterminent notre existence à tel point que l'art et la théorie de notre époque se voient à présent forcés de jeter sur internet un regard distant, hors ligne, qui vacille entre suspicion, fétichisation et ironie, capable de réfléchir sur les conditions de possibilité des réseaux, sur les architectures internes et externes d'internet - et de ses diverses ramifications existentielles - qui, en s'infiltrant dans nos vies à tant de niveaux, devient presque «invisible» (Chatonsky 2015). 
10), aujourd'hui ce sont la circulation, la viralité, le remixage et l'utilisation des contenus qui leur donnent un sens, une valeur et une forme. Un mème est coréalisé partout et par tout le monde : «chacun, anonymement, laisserait sa trace dans des nappes de discours sans auteur», a écrit l'historien Roger Chartier à propos du texte électronique (Chartier). Or ce cinéma démocratisé et bon marché auguré par Mekas est-il de nos jours un fait accompli? En 2017, 400 heures de vidéo furent téléchargées par minute sur YouTube: ces millions d'heures de contenus vidéo sont-elles du cinéma ou autre chose? Quels liens entretiennent-elles avec le médium cinématographique - médium qui, jusqu'à la moitié $\mathrm{du} \mathrm{XX}^{\mathrm{e}}$ siècle, dominait la production d'images en mouvement?

Les problématiques qui vont être ici développées sont animées par cette nouvelle catégorie d'images en mouvement nommée «vidéo vernaculaire». Dans un premier temps, nous tenterons de définir, ou du moins de contextualiser l'apparition et la popularisation du concept de vidéo vernaculaire dans la littérature scientifique anglophone et francophone. Il s'agira ensuite d'essayer d'en dresser les traits caractéristiques et de nous demander si, comme la vidéo, le cinéma peut être vernaculaire ${ }^{2}$, et, si c'est le cas, quelles sont les conditions de son existence. Dans quelle mesure le vernaculaire pourrait-il constituer un genre cinématographique? En quoi le régime visuel du vernaculaire renouvelle-t-il les régimes d'expression du cinéma? Quels risques, quelles limites, quelles contradictions en découlent? Afin de répondre à ces questions, nous exposerons et analyserons des œuvres filmiques qui nous aideront à méditer la possibilité d'une artialisation contemporaine du vernaculaire. Ces œuvres, résultat du réemploi cinématographique de vidéos vernaculaires, témoignent d'actes d'appropriation réalisés par des porteurs d'autorité artistique et dont nous relèverons, pour finir, les enjeux politiques et esth-éthiques.

\section{Le sort du concept en contexte académique}

Depuis quelques années déjà, le qualificatif de vernaculaire appliqué aux vidéos amateurs et domestiques qui prolifèrent sur le web est employé dans la recherche universitaire, où l'intérêt pour les

Bien que son approche soit éloignée de la nôtre, il faut ici mentionner l'un des rares textes qui utilisent le terme «vernaculaire» appliqué au cinéma, l'article de Miriam Bratu Hansen «The Mass Production of the Senses: Classical Cinema as Vernacular Modernism» (1999). L'autrice y propose d'étendre l'étude de l'esthétique moderniste aux pratiques culturelles qui ont à la fois articulé et médiatisé l'expérience de la modernité, tels que les phénomènes produits et consommés en masse que sont la mode, le design, la publicité, l'architecture, la photographie et le cinéma. Elle qualifie ce type de modernisme de « vernaculaire» parce que «le terme vernaculaire combine la dimension de l'usage quotidien, avec les connotations du discours, de l'idiome et du dialecte, avec la circulation, la promiscuité et la traductibilité». Selon Hansen, le cinéma classique d'Hollywood pourrait être imaginé comme un modernisme vernaculaire produit industriellement, en masse. 
formes audiovisuelles créées par les utilisateurs non-professionnels d'internet est croissant. Ces formes audiovisuelles se sont propagées et démultipliées surtout depuis l'apparition de YouTube en 2005. La vidéo vernaculaire est désormais accessible en ligne, sur des plateformes d'hébergement vidéo, ou sur des applications de téléphone mobile et de live-streaming comme TikTok, Twitch ou Snapchat. Elle redéfinit les notions de domesticité ou d'intimité en effaçant les frontières entre le public et le privé, l'extime et l'intime. Elle rend également poreuses celles qui différencient l'artiste, le professionnel et l'amateur. En 2006, une année après la naissance de YouTube, le magazine américain Time choisit «You» comme la personnalité de l'année. Par ce geste audacieux, le magazine faisait référence à YouTube, mais aussi à Wikipedia, Myspace, Facebook, et globalement, à l'éclosion du web participatif ou web 2.0. L'individu lambda, ordinaire, le produser ou prosumer ${ }^{3}$ - néologismes qui combinent le rôle de producteur et celui de consommateur-utilisateur - et son User-Generated Content s'étaient placés au centre névralgique de la culture planétaire ${ }^{4}$. La vidéo serait dorénavant le «médium du peuple [...] la forme vernaculaire de cette ère» (Sherman 161).

Tout d'abord, dressons un historique sommaire des usages de l'adjectif «vernaculaire», tel qu'il est associé à la vidéo. C'est avec la naissance et l'essor de YouTube qu'a lieu la popularisation du concept. C'est probablement en 1995 que l'expression vernacular video apparaît pour la première fois, dans «Vernacular Video: For the Growing Genre of Camcorder Journalism, Nothing Is Too Personal», article de la professeure de communication Patricia Aufderheide. L'autrice, en conversation avec Ed Fouhy, directeur du Pew Center for Civic Journalism, y examinait la manière dont le tournant numérique et ce qu'elle nomme «journalisme de caméscope» affaiblissaient le rôle traditionnel du journaliste comme intermédiaire ayant accès à l'information et la traitant à destination de la population. Une décennie plus tard, en 2006, Judi Hetrick, professeure de journalisme, publie l'article «Amateur Video Must Not Be Overlooked». Elle y recommande de préciser les modalités de catégorisation des vidéos amateurs et propose la création d'un nouveau genre constitué de «vidéos communautaires vernaculaires » dont elle préconise l'archivage. Hetrick défend dans ce texte la valeur de la vidéo vernaculaire comme source précieuse de documentation sociale. Il apparaît donc que, dans un premier temps, le terme «vernaculaire» est associé à des problématiques propres au champ du journalisme.

3. Le néologisme «prosumer» fut inventé en 1980 par le futuriste américain Alvin Toffler (1980). Il fut ensuite repris, entre autres, par George Ritzer et Nathan Jurgenson (2010). Axel Bruns proposa les termes «produser» et «produsage» (Bruns 2008).

4. Le sous-titre utilisé par la revue fut «You control the Information Age. Welcome to your world», et la couverture incluait un écran d'iMac dont la matière réfléchissante faisait apparaître sur l'écran de l'ordinateur le visage de tous ceux qui prenaient le magazine entre leurs mains. 
Or, à la même période, les travaux issus de la discipline naissante des YouTube Studies achèvent de consolider l'expression «vidéo vernaculaire» dans le cadre d'analyses plus précises croisant les études culturelles, visuelles et des media. La thèse de Jean Burgess soutenue en 2007 et portant sur ce qu'elle nomme la «créativité vernaculaire» puis son livre co-écrit avec Joshua Green et publié deux ans plus tard, Youtube, Online Video and Participatory Culture, furent pionniers dans cette consolidation. Suivront «Vernacular Video», article de Tom Sherman et publié en 2008 dans l'anthologie fondatrice Video Vortex Reader: Responses to YouTube; puis, en 2009, l'ouvrage collectif The YouTube Reader, où l'emploi du qualificatif reste tout de même assez marginal (Snickars et Vondereau). Il sera pareillement employé par Robert Glenn Howard pour étudier le «web vernaculaire» dans son article «The Vernacular Web of Participatory Media»-néanmoins, l'auteur y fait référence à des formes principalement textuelles du web participatif (blogs, forums, commentaires) à la lumière de l'analyse du discours et de la critique rhétorique, et non pas à des formes vidéographiques. De 2005 à 2010, le concept de vidéo vernaculaire est ainsi de plus en plus utilisé sans qu'il y ait cependant un recours systématique à ce qualificatif et qu'une théorisation organisée et approfondie soit engagée pour définir ce qu'il signifie en vidéo. Burgess utilise le concept de créativité vernaculaire afin d'étudier des pratiques créatives quotidiennes et ordinaires qui voient le jour indépendamment des valeurs légitimes, hégémoniques, de la haute culture et en dehors des limites symboliques des mondes artistiques institutionnalisés. Les exemples qu'elle donne relèvent de la photographie et du film de famille, du scrapbooking, de la tenue d'un journal intime ou du vidéo-blog, etc ${ }^{5}$. Ce sont des pratiques populaires qui restent liées à la culture hégémonique : selon Burgess, la créativité vernaculaire fonctionne souvent en référence aux valeurs, à l'esthétique et aux techniques des professions créatives et des mondes artistiques établis. Elle pourrait être apparentée à un pastiche reposant sur l'absorption d'éléments de la culture populaire et de la culture savante sous le mode du synchronisme culturel ${ }^{6}$. Cette créativité vernaculaire préexiste bien entendu à l'ère numérique, mais elle a été bouleversée par l'irruption de nouveaux médias et, en leur sein, de nouvelles configurations de production culturelle. Même si chaque exemple de créativité vernaculaire participe d'une mise en commun, il « est une représentation d'une vie spécifique, d'un temps spécifique et d'un lieu spécifique» (Jenkins 2007), sa remédiation numérique

En France, dans la même lignée que Burgess, nous pouvons citer les travaux de Laurence Allard, qui parle d'«individuation expressive», " vidéoludisme» et «créativité ordinaire» pour qualifier nos usages du téléphone mobile connecté, dispositif qu'elle considère, reprenant les termes de Foucault, comme une «technologie du soi/pour soi». (Allard 2014a ; Allard 2017).

6. À cet égard, Richard Hoggart a forgé la notion d'attention oblique pour décrire la modalité d'attention sélective, d'appropriation et de réception réticente des classes populaires ou populations démunies des instruments culturels hégémoniques. Cette attention est faite d'un mélange de distance et de méfiance vis-à-vis de la culture dominante, c'est-à-dire, des contenus produits par les médias de masse (Hoggart). 
permettant l'obtention d'une «citoyenneté culturelle» (Burgess 248), celle-ci étant comprise comme une «pratique de participation active à la sphère publique culturelle» (250).

L'article du vidéaste Tom Sherman, «Vernacular Video», constitue un autre texte clé pour comprendre les rapports complexes entre art et vernaculaire. Selon Sherman, afin de se distinguer de la prolifération de la vidéo vernaculaire, l'art vidéo institutionnalisé s'est davantage rattaché à l'histoire des arts visuels et du cinéma, en tournant le dos à «ses potentialités en tant que médium de communication» (162). L'art vidéo est durement critiqué par Sherman, qui le considère comme conservateur et barricadé dans le confort de l'espace muséal. En tant qu' «édition limitée» ou objet physique unique et fétichisé, il serait anachronique, car il ne lancerait aucun défi aux raisons d'être de l'institution muséale, ne ferait qu'imiter les formes d'art précédentes comme la peinture ou la sculpture, et contribuerait à renforcer la valeur auratique, l'authenticité, l'ici et le maintenant, dirait Walter Benjamin, de la collection de l'institution.

En 2013, le chercheur et réalisateur Peter Snowdon fait un apport théorique crucial. Dans «The Revolution Will Be Uploaded: Vernacular Video and the Arab Spring», il analyse les vidéos du printemps arabe qu'il qualifie d' «anarchive vernaculaire», donnant à la catégorie du vernaculaire, jusque-là uniquement descriptive, une nouvelle nuance plus politique, basée sur l'utilisation du terme proposée par Ivan Illich au début des années 1980. Illich, dans Le Genre vernaculaire et Le Travail fantôme, identifia les pratiques vernaculaires comme des activités centrales dans la vie communautaire, qui contribuent à la rendre autonome à la fois du marché et de l'État, entendus comme des instances de pouvoir. Le vernaculaire entraîne la réduction des transactions monétaires et favorise l'expansion de formes de subsistance extérieure à l'économie capitaliste. De même, il est pour Illich incompatible avec une vision possessive du monde. La pensée scientifique occidentale, selon Illich, nie les registres d'expérience vernaculaires en les reléguant au domaine du privé ou du subjectif. La disparition du genre vernaculaire serait ainsi «la condition déterminante de l'essor du capitalisme» (Illich 2005, 251). Le vernaculaire est donc avant tout le premier domaine de résistance des peuples à la colonisation de leurs formes de vie quotidiennes par l'État ou le marché capitaliste.

Pour Snowdon, le vernaculaire étant «la mise en commun de ce qu'il y a d'unique et fragile en chacun de nous », celle-ci «rend possible l'invention de nouvelles formes» (422). En revanche, la plupart des utilisations individualistes occidentales de la vidéo en ligne, que le monde académique qualifie de vidéos vernaculaires, doivent être appréhendées, selon le réalisateur, comme du travail fantôme, une «forme de servitude non rétribuée [qui] ne contribue nullement à la subsistance» (Illich 2005, 202), du «travail non payé dont l'accomplissement permet précisément que des salaires soient payés» (202). La plus grande partie de ces pratiques vidéo contribuent peu ou pas au renforcement des formes d'autonomie et de subsistance collectives de nature communautaire mais permettent aux 
plateformes d'obtenir des profits. Développons à présent cette piste afin de considérer dans quelle mesure les vidéos vernaculaires sont des modalités de travail numérique, immatériel, du digital labor ${ }^{7}$, effectué par les utilisateurs au bénéfice des plateformes de partage et sur la base d'une forme d'exploitation capitaliste acceptée en échange d'un supplément de visibilité. De la même manière, lorsque des artistes et des cinéastes s'approprient ces contenus audiovisuels pour créer des œuvres d'art, ne mettent-ils pas en place également des modes d'exploitation au sein du binôme artiste - produser, au détriment de l'utilisateur?

Selon Snowdon, les vidéos du Printemps arabe, par leur nature à la fois anonyme et collective, constituent un bien commun et apparaissent comme «un premier pas vers l'invention de la vidéo en ligne comme une pratique véritablement vernaculaire» (Snowdon 411). Pour lui, le fait que le nom et l'identité des vidéastes soient très souvent négligeables ou que ceux-ci adoptent des pseudonymes apparente ces vidéos aux mots d'une langue vernaculaire plutôt qu'à des énoncés d'auteurs spécifiques prononcés ou écrits dans la langue véhiculaire. Le vernaculaire en vidéo est donc bien le propre des copistes et des troubadours.

Sur la base de ces travaux liminaires, il apparaît que la notion de vidéo vernaculaire est désormais opératoire, en particulier dans le monde académique anglophone, et malgré l'hétérogénéité de ses usages. En France, les quelques chercheurs qui s'en servent empruntent la voie politique de Snowdon et l'adoptent dans des analyses de vidéos de mobilisations, soulèvements et révolutions, notamment celles du Printemps arabe. Il s'agit essentiellement de Dork Zabunyan, de Cécile Boëx et d'Ulrike Lune Riboni. L'adjectif est aussi très succinctement mobilisé par Giuseppina Sapio et Beatriz Rodovalho pour décrire l'esthétique des films de famille, cette fois-ci dans le sens plus dépolitisé de l'amateurisme et du domestique comme dans les études sur le web participatif et consacrées à YouTube.

\section{Vidéo vernaculaire : identification d'une catégorie visuelle}

De quoi la vidéo vernaculaire est-elle le nom? En raison de son incommensurabilité, elle est difficilement gérable, classifiable, analysable d'un point de vue esthétique, social ou culturel, notamment par les théoriciens des images ou des medias ${ }^{8}$, ou encore transformable en archive historique du contemporain. La masse, l'hétérogénéité, l'aspect parfois éphémère - les vidéos en ligne peuvent disparaître

Pour une étude et théorisation du digital labor, voir Casilli (2019).

Nous ne voulons aucunement dire par là que cette masse audiovisuelle ne soit pas classée et ordonnée dans l'espace virtuel: les algorithmes remplacent le «principe archontique» ordonnant et propre à toute archive (Derrida 1995) dans 
si leurs auteurs ou la plateforme le désirent -, non-standardisé, circonstanciel et fragile de ces images compliquent leur analyse et les tentatives d'en établir une définition, de dresser des tendances, des sousgenres, et d'en cerner les enjeux politiques et socioculturels. Jusqu'à présent, l'essai de Snowdon a été le seul à proposer la construction d'une définition précise, à travers son exercice de resémantisation du concept. Du reste, il semblerait que l'expression soit devenue une étiquette englobant un ensemble de pratiques et de formes vidéo très hétéroclite et dont l'ampleur ou l'inexactitude pourrait porter atteinte à la valeur d'usage du concept.

Orientons-nous vers un media proche de la vidéo pour y voir plus clair théoriquement. Clément Chéroux est l'un des principaux penseurs contemporains de la photographie vernaculaire, qu'il définit essentiellement par trois traits : utilitaire, domestique et hétérotopique (2013). De plus, le vernaculaire n'est pas destiné à la commercialisation mais à la consommation personnelle, c'est «l'envers de la marchandise industrielle» (10). À ce propos, il cite Pierre Frey, qui affirme que le vernaculaire est «tout ce qui demeure périphérique ou extérieur aux flux mondiaux du capital» (10). Cette définition fait écho à celles de Snowdon et d'Illich opposant le vernaculaire à la marchandise, au capitalisme, et écarte immédiatement YouTube ou les salles de cinéma des espaces du vernaculaire. Il est intéressant que Chéroux oppose également le vernaculaire à l'art, ce qui semble situer l'art et la marchandise du même côté. La photographie vernaculaire n'est pas artistique : elle est «l'Autre de l'art» (13), occupant une position d'altérité, de «miroir de l'art» (14), permettant ainsi «de le redéfinir» (19). Contrairement à l'art, la photographie vernaculaire est considérée comme «sans qualité et sans rareté» (13) - produite «sans vouloir artistique» (13) mais évidemment pas dénuée de qualités esthétiques. Finalement, une image vernaculaire, si un jour elle fut utilitaire, «ne sert plus ce pourquoi elle avait été originalement produite» (13) : cette perte de la valeur d'usage montre à quel point pour Chéroux le rôle des artistescollectionneurs du vernaculaire est primordial. En effet, sans les découvertes réalisées par ces artistes, la force poétique de la photographie vernaculaire serait restée dans l'ombre des coffres et des caves. La réappropriation de ces images les soustrairait à leur valeur d'usage - «une photographie utilitaire ne devient réellement vernaculaire qu'à partir du moment où elle perd sa valeur d'usage initial» (13). Les artistes dévoilent ainsi la puissance critique, anthropologique et plastique de la photographie vernaculaire: «véritables découvreurs du vernaculaire, les artistes en sont aussi les grands promoteurs 》 (17). Toutefois, l'analyse de Chéroux semble ici paradoxale, puisqu'il semblerait que c'est l'artiste qui produit le vernaculaire en s'en emparant, alors même que le vernaculaire est l'Autre de l'art.

la tâche d'identifier, classifier et déterminer la visibilité des contenus des bases de données du net. Voir aussi Rouvroy et Berns. 
Examinons alors si la vidéo vernaculaire est bien utile, domestique et hétérotopique. L'apparition de la notion en même temps que des plateformes d'hébergement en ligne laisse supposer une corrélation entre la vidéo vernaculaire et l'essor du web 2.0, avec ce qu'il implique de mise en ligne, de partage et de circulation. Si, bien avant l'essor du web participatif, ce type de vidéo existait déjà, il était qualifié de «vidéo amateur», et était ainsi inscrit dans la continuité du cinéma domestique, dont le rôle premier est celui des images-souvenirs. À l'inverse, la vidéo vernaculaire est majoritairement produite à des fins communicationnelles et elle n'assure pas comme le film de famille la fonction d' «instrument de mémoire» (Odin 51). L'utilité de la vidéo vernaculaire résiderait dans sa nature relationnelle, «conversationnelle» (Gunthert), Laurence Allard allant jusqu'à parler du «tournant conversationnel» de la vidéo sur Facebook (Allard 2014b). La vidéo vernaculaire sert à transmettre aux autres un message, une information, une expérience, une émotion, et par ce biais à tisser des relations avec d'autres membres d'une communauté (virtuelle), à déloger le solipsisme. Parallèlement, Allard souligne les phénomènes de «narration de soi» et d' «individuation» qui traversent cette vidéo conçue comme une «technologie du soi». Or cette expressivité du soi n'est pas égocentrée mais intersubjective, et ne saurait se passer de l'Autre, et si la «voix intérieure» s'exprime, c'est toujours - admettant l'insuffisance ontologique de la condition humaine - en attente d'une voix d'autrui qui donne la réplique et d'un regard qui reconnaisse et retrouve le nôtre. La vidéo vernaculaire n'est jamais une île, mais un mot énoncé en attente d'une réponse, une bouteille lancée avec espoir à une eau virtuelle peuplée, un nœud d'interaction sociale.

Par ailleurs, considérer la définition de Chéroux, au même titre que d'autres auteurs et au regard d'autres disciplines, incite à penser que le vernaculaire s'affirme en négatif de l'institutionnel, de l'officiel, du savant, de l'élitiste. Le vernaculaire n'émerge que de manière dialectique; contre ou face à un contraire, il s'appuie sur l'existence d'institutions dont il faut le distinguer pour montrer sa nature autre. Le vernaculaire se convoque par rapport à un centre, en fonction duquel il est nécessairement décentré. Spécifiquement, la vidéo vernaculaire s'oppose au cinéma et à la télévision, en tant que deux continents visuels, deux syntaxes canoniques et deux instances de pouvoir industrialisées, commerciales, artistiques ou professionnelles. «Broadcast yourself», le slogan initial de YouTube faisait directement référence à la télévision : à la centralité de ce media emblématique du support vidéo s'est opposée inévitablement la décentralisation rhizomatique que promettait internet, très vite devenu l'écosystème par excellence de la vidéo vernaculaire ${ }^{9}$. Le bouleversement discursif, qui correspond à la prise de parole d'un public préalablement silencieux, a contribué au fait qu'internet soit devenu un

La télévision fonctionne sur le principe d'une offre de contenus limitée du haut vers le bas, d'experts-auteurs pour une multiplicité de spectateurs, alors qu'internet est basé sur une énonciation horizontale, une auctorialité partagée et atomisée et des destinataires indéterminés, ce «public invisible» de danah boyd (2008). 
espace privilégié d'expression pour les voix subalternes et dissidentes. Par la suite, ce bouleversement l'a conduit à incarner l'élément majeur d'érosion de valeurs éthiques et politiques telles que l'objectivité ou la vérité - la post-vérité (McIntyre), intrinsèquement liée à la montée en puissance du cyberespace. Par ailleurs, s'il a rendu possible de nouveaux modes d'organisation, de création, de production ou de coopération au sein de la société civile, l'espace d'internet ne constitue pas une terre rhizomatique déterritorialisée et décentralisée: «la plupart des infrastructures médiatiques que nous utilisons sont entre les mains de quelques entreprises, ce qui rétablit l'ancien modèle de domination des médias de masse» et «la décentralisation des moyens de production s'est accompagnée d'une centralisation des relations de production» (Apprich 136).

La domesticité évoquée par Chéroux pose problème. Henry Jenkins, dans son ouvrage Convergence culture sur la culture participative, préfère au terme vernaculaire celui de «grassroots creativity », la créativité de la base, expression qui, comme le mot vernaculaire, fait référence à un ancrage de terrain (Jenkins 2006), ce qui n'empêche pas que, avec le tournant numérique, le vernaculaire se soit émancipé de la terre pour s'envoler dans les nuages, le cloud, à travers une connectivité globale. Si le milieu d'existence contemporain de la vidéo vernaculaire est le web, avec ce qu'il comporte d'équipements tout à fait matériels - câbles transnationaux, serveurs, etc. -, force est donc de constater que celle-ci déborde depuis longtemps l'espace domestique, en s'ouvrant à une circulation et à une visibilité de niveau planétaire. Si à l'origine, la plupart des vlogs étaient enregistrés à la maison grâce à une webcam et si l'espace originaire du YouTubeur était la chambre, les caméras se sont depuis émancipées de l'ordinateur de bureau. Aujourd'hui, la vidéo vernaculaire est produite partout et par tout le monde, Mekas dixit, et l'ère des home movies projetés exclusivement en contexte familial ou en cercles restreints d'amis est terminée ${ }^{10}$. Alors que le vernaculaire définit traditionnellement ce qui provenait de la maison, c'est la globalisation, l'hyper-connectivité et la circulation délocalisée qui ont fait exploser la production et la diffusion de la vidéo vernaculaire. Cet écart sémantique est déjà contenu dans la définition de la «vidéosphère» que propose Régis Debray en 1992, la vidéosphère étant l'ère médiatique dominée par le visuel. En opérant une distinction entre l'idole, propre à la période antérieure à l'invention de l'imprimerie, l'art, emblème du monde post-imprimerie, et le visuel, né avec la vidéo,

10. Nous ne voudrions pas laisser entendre par-là que de nos jours tout individu produit des images en mouvement, il va de soi qu'une grande partie des utilisateurs d'internet n'envoie et ne publie pas de vidéos en ligne mais se contente de les consommer. Par ailleurs, Ulrike Lune Riboni (2019) insiste sur l'idée qu'image anonyme n'est pas synonyme d'image privée. Aujourd'hui, les albums de famille sont publics et partagés, mais ces stratégies de partage sont diverses et représentent différentes strates de «publicité». Les dimensions familiale et sociale dans lesquelles les conditions de visibilité sont restreintes au cercle d'amis, famille et amoureux coexistent avec celle de la construction individuelle de l'identité sociale, du récit de soi et des pratiques expressivistes qui peuvent avoir des dimensions totalement publiques. 
Debray écrit: «L’idole est autochtone, lourdement vernaculaire, enracinée dans un sol ethnique. [...] Le visuel est mondial (mondiovision), conçu dès sa fabrication pour une diffusion planétaire» (289). Il existe au sein de la vidéo vernaculaire une forte tension entre, d'une part, des modes de vie coutumiers et idiomes communautaires et, d'autre part, des pratiques médiatiques qui les déterminent, les multiplient et les diffusent, étendant ainsi leur résonance au-delà de leur actualité et localité d'origine. Une des conséquences de cette extension porteuse d'uniformisation culturelle croissante à l'échelle mondiale pose dès lors question: dans quelle mesure le «village global» (McLuhan 31) du web 2.0 et les architectures des applications et plateformes provoquent-ils la désagrégation et l'effacement des différences, des singularités et des particularités propres au vernaculaire ${ }^{11}$ ?

\section{De la vernacularisation à l'artialisation : transformations du vernaculaire}

À l'instar de la pellicule argentique lorsque le Super 8 s'est démocratisé dans les années 1960 en tant que format domestique par excellence, le médium vidéo a lui aussi connu un processus de vernacularisation, radicalement accéléré avec le passage au numérique ${ }^{12}$. Après ce premier processus de vernacularisation, un phénomène d'artialisation de la vidéo vernaculaire se développe à partir des années 2010. La vidéo vernaculaire, comme jadis le film de famille amateur, s'installe au sein de l'espace muséal et de la programmation des plus grands festivals de cinéma en étant intégrée dans des œuvres d'art reconnues, médiatrices, prescriptrices. Avec ces films d'une nature hybride, utilisant le vernaculaire pour en faire de l'art, s'affirme le paradoxe qui anime le titre oxymorique de cet article: le vernaculaire comme genre cinématographique. Mentionnons quelques exemples d'œuvres, qui réemploient et s'approprient des vidéos vernaculaires trouvées en ligne: le film Roman National (2018) de Grégoire Beil, réalisé à partir de vidéos de jeunes Français trouvées sur l'application Périscope, a été montré pour la première fois au festival Cinéma du Réel où il reçut la Mention Spéciale du Jury,

11. À propos de cette problématique, nous nous permettons de renvoyer à notre chapitre «Contes d'été virtuels. Le surcyclage de la vidéosphère dans Roman National (2018) de Grégoire Beil» (Hernández López).

12. Notons encore une fois que cette production pré-internet était souvent désignée par le terme «amateur». Le fait de préférer ce mot à celui de «vernaculaire» s'explique probablement par le fait que dans le domaine du vernaculaire les liens de parenté doivent être évacués. Comme le pointe Chéroux, on ne dit pas «mon album de photos vernaculaires» car le vernaculaire est le royaume des inconnus. Ce sont «seulement les enfants [...] des autres qui nous apparaissent» comme vernaculaires (Chéroux 14). En outre, le terme «amateur» a une certaine connotation négative que nous trouvons problématique, car situé en dessous du professionnel, avec un manque de qualité, de ressources, d'exigence ou de productivité par rapport à celui-là. L'amateur est socialement stigmatisé et surdéterminé, mais pas le vernaculaire, notion encore trop peu répandue pour être connotée. 
avant d'être diffusé au Palais de Tokyo lors de l'exposition L'Ennemi de mon ennemi de l'artiste et producteur du film Neïl Beloufa. Le documentaire Present. Perfect. de Shengze Zhu (2019), composé de vidéos de plateformes chinoises de streaming en direct, a gagné le premier prix au festival de cinéma de Rotterdam. Going South de Dominic Gagnon (2018), réalisé avec des vidéos YouTube, eut sa première dans le festival suisse Visions du Réel. Les films cités forment un cinéma vernaculaire en ce qu'ils font collection du vernaculaire. Comme si on était passé de l'âge des chasseurs d'images à celle des collecteurs (Fontcuberta 178), ces œuvres font archive du vernaculaire, dans un travail de documentation qui permet son analyse critique et le dévoilement de ses puissances affectives, culturelles et politiques. Si en raison de leur nature, les plateformes majoritaires contredisent a priori le potentiel de résistance ou de transgression que comporte une partie de la vidéo vernaculaire - tout en participant de sa diffusion -, l'art peut trouver, précisément dans cette faille, un point d'intervention. Tout comme le montre Chéroux dans le domaine de la photographie, ces réalisateurs jouent un rôle fondamental en pointant du doigt, retrouvant, sélectionnant, exhumant, réactivant cette (an)archive, cette mémoire audiovisuelle vernaculaire, à laquelle ils donnent une nouvelle visibilité. Ils détournent, déjouent et subvertissent ainsi les stratégies d'éditorialisation, de suggestion, de modération, de hiérarchisation des contenus des réseaux sociaux et des plateformes. Ces cinéastes sont, en fait et surtout, les spectateurs de ces images, puis des prescripteurs de sens, qui révèlent des valeurs jusque-là cachées, sublimant les vidéos vernaculaires pour leur faire dire leurs secrets, merveilleux et atroces, et actualisant leurs virtualités. En les adoptant, ils enrayent le flot visuel massif et continu du cyberespace, et ils célèbrent l'esprit et la singularité, plutôt que la maîtrise et la compétence technique. En les nouant par des liens inédits et dialectiques, ils instaurent de nouvelles constellations et mettent en marche ces processus de singularisation des images, qui deviennent, une fois resémantisées, à nouveau et autrement significatives.

Trailer de Present. Perfect.

https://vimeo.com/309692589. ZHU, Shengze, réal. Present. Perfect. Burn the Film, 2019.

Quelles sont donc les conséquences de cette appropriation par les circuits de l'art de la création vernaculaire reconnue par les instances de légitimation épistémologique et socio-culturelle que sont les musées, festivals de cinéma, etc. ? D'abord, les institutions qui programment, diffusent, soutiennent et récompensent ces œuvres ajoutent du crédit symbolique et culturel à cette esthétique de la vie ordinaire et aux artistes qui y prêtent attention, souvent avec un regard qui oscille entre fascination et critique. Les vidéos remontées, qui ne sont modifiées en profondeur ni dans leur forme ni dans leur contenu, mais le sont notamment dans leurs significations, leurs affects et leur durée, sont la matière première du travail de recyclage des réalisateurs et deviennent partie intégrante d'œuvres artistiques qui génèrent un bénéfice économique et symbolique. Il est indéniable que ces auteurs profitent de ces représentations 
vernaculaires pour se faire une place dans le monde de l'art - ce qui n'empêche pas qu'ils aient un vrai intérêt pour ces images et qu'ils s'en emparent pour construire une représentation subjective des réalités contemporaines. Si le vernaculaire n'est ni art ni marchandise, ces vidéos ont-elles cessé de l'être au moment de leur projection publique, alors que les producteurs et artistes qui les ont intégrées à leur film ont été potentiellement rétribués sur la base de droits de diffusion, et qu'elles sont devenues par-là simultanément art et marchandise? Or, selon Chéroux, le vernaculaire doit perdre sa fonction originelle et apparaît quand l'objet est déplacé ailleurs, décontextualisé.

In fine, il n'est plus possible de penser le vernaculaire sans réfléchir à des notions comme celles d'artiste, de propriété intellectuelle ou de droit d'auteur. Le vernaculaire, s'il s'extrait des logiques et des injonctions du marché capitaliste, doit aussi fuir celles qui sont liées à la propriété productive, à la valeur iconomique (Szendy) des œuvres. Comment parvient-on à intégrer cet aspect dans la définition du vernaculaire tout en respectant les droits légitimes des artistes et cinéastes? Il s'agit là d'un débat complexe qui engage à questionner les relations de subordination et de domination qu'inclut le concept de vernaculaire, plus encore quand il qualifie un objet approprié: une question de proximité et de distance géographiques, sociales, culturelles, économiques, idéologiques, intellectuelles, etc., entre l'artiste et les auteurs des images-sources; une question de transparence et d'opacité, c'est-àdire de modalités esthétiques et discursives dans lesquelles les voix et les significations présentes au sein de l'œuvre d'art sont entrelacées, réduites au silence, modifiées ou amplifiées, ces deux dernières actions étant appréhendées différemment dans chacune des œuvres d'art mentionnées. En ce sens, le photographe Joan Fontcuberta propose de substituer au terme d'appropriation celui d'adoption: si le premier fait référence à un geste privé, le second est une déclaration publique, équivalant à «reconnaître de manière publique une valeur symbolique, faire profession d'une attitude envers le prochain» (60). Il n'y a pas de dépossession dans l'adoption, mais uniquement une élection, une sélection: «on ne réclame pas la paternité des images, uniquement leur tutelle idéologique» (60). Le caractère commun du vernaculaire refait surface: est commun ce qui est inappropriable, mais aussi adoptable - ce qui suppose d'octroyer « un caractère officiel et visible à la lecture que quelqu'un fait» d'une image (60). Dans les pratiques d'adoption des images considérées comme biens communs, la propriété ne relève pas d'une appropriation ou d'une privatisation, mais de l'usage que chacun fait de l'image: «la qualité artistique ne réside plus dans l'acte de la production mais dans l'acte intellectuel de prescription des valeurs que les images peuvent accueillir ou contenir: des valeurs sous-jacentes ou qui leur ont été injectées » (54). En partant du concept d'adoption de Fontcuberta, nous en venons donc à considérer que le vernaculaire n'est pas incompatible avec des pratiques d'adoption artistique intégrées au marché de l'art et à l'industrie du cinéma, à condition que le geste d'adoption respecte le caractère commun et inappropriable du vernaculaire, c'est-à-dire, que les vidéos, une fois insérées dans l'œuvre d'art, 
soient restituées, partagées avec les vidéastes qui les ont créées. Ceci peut se faire tantôt en rendant accessible l'œuvre de l'artiste sur les plateformes numériques où les vidéos-source ont été trouvées, tantôt en donnant aux vidéastes l'accès à l'œuvre produite - ce que les réalisateurs ne font pas systématiquement, par exemple Grégoire Beil ou Dominic Gagnon ${ }^{13}$.

En effet, il nous semble dangereux d'affirmer, comme le fait Fontcuberta, que ces images sont anonymes, «muettes» et «orphelines» $(152)^{14}$. Les vidéos vernaculaires ont été filmées par des individus qui s'y expriment. Ce que fait l'artiste-recycleur, c'est d'abord d'accorder à ces vidéos une attention soutenue, patiente et fascinée, celle du spectateur de cinéma, alors que l'économie attentionnelle de la vidéosphère se caractérise par un regard distrait. Son travail plastique et discursif fait parler ces vidéos autrement - vidéos qui, parfois, deviennent la matière première de leur propre travail critique. De leur côté, les circuits institutionnels de l'art et des festivals de cinéma permettent de donner un autre espace de visibilité à ces objets visuels et ces voix ; ils travaillent avec les artistes contre les moteurs de recherche et les algorithmes des réseaux socio-numériques qui rendent invisibles ces contenus. Or, le terme d'anonyme reste problématique en ce qu'il efface l'individu à l'origine de ces contenus. En considérant ces vidéos comme anonymes, on les rend plus appropriables, comme si elles nécessitaient une signature, une subjectivité d'artiste pour leur conférer une voix. Si nous défendons le droit au détournement, à la copie et au copyleft, nous ne pouvons ignorer les rapports de pouvoir qui s'installent entre le sujet-vidéaste et le sujet-copiste dans le cas des projets photographiques étudiés par Fontcuberta - sous le nom de post-photographie - ainsi que dans les films mentionnés ci-dessus.

La référence concrète à la voix et aux espaces discursifs est ici essentielle et concerne ces pratiques artistiques directement. Comme le souligne Gayatri Chakravorty Spivak dans «Can the Subaltern Speak?», la portée des mots est surdéterminée par la position dans l'échelle économicosociale. Une partie des vidéos adoptées/appropriées dans ces œuvres est réalisée par des sujets politiques subalternes. Ces films articulent-ils les voix des autres notamment d'individus appartenant à des minorités? Permettent-ils aux personnes qui se filment de développer un espace discursif en tant que sujets politiques? En augmentant la visibilité de ces images, augmentent-ils les échos et les répercussions de leurs mots, fonctionnant telle une caisse de résonance, ou, au contraire, font-ils entendre uniquement la voix des cinéastes?

13. Même s'ils mentionnent dans le générique de fin les comptes en ligne des vidéastes (Beil) ou le nom de la vidéo dans la plateforme d'hébergement (Gagnon), ces réalisateurs n'ont pas directement rentré en contact avec les personnes qui ont filmé les images de leurs films, ni rendu leurs films disponibles en ligne.

14. André Gunthert a examiné la pénétration des contenus anonymes dans l'espace médiatique traditionnel (Gunthert 2018). Lui aussi qualifie la masse d'images de la conversation numérique de vernaculaires, conversationnelles, «autoproduites » et «anonymes». 
Bande-annonce de Going South.

https://www.youtube.com/watch?v=p9LL-fhSVKU. GAGNON, Dominic, réal. Going South. Dominic Gagnon, 2018.

Les «voix vernaculaires» (Hauser) et le «discours vernaculaire» (Ono et Sloop) peuvent être identifiés aux voix effacées, déplacées dans les marges, ces contre-discours marginalisés face aux voix hégémoniques. Bien qu'elles reposent sur une signature et l'identification d'un auteur, ces pratiques d'appropriation, ou, dans le meilleur des cas, d'adoption peuvent s'insérer dans la longue histoire des pratiques artistiques de collaboration, qui sont en totale harmonie avec le zeitgeist contemporain qui s'exprime à travers les notions de détournement, de mort de l'auteur, de Creative Commons et de culture du remix à l'ère post-internet dans laquelle tout un chacun est simultanément spectateur et créateur. Pour ce faire, et être complètement en accord avec cette position fortement politique, le cinéma vernaculaire doit négocier les modalités d'adoption des contenus avec la communauté qui les a produits, afin que l'adoption ne se transforme pas en oppression et domination socioculturelle.

D’un autre côté, contrairement à Tom Sherman qui soutient que la vidéo vernaculaire se caractérise par une «anesthétique», une «absence de conscience esthétique» (163), nous croyons qu'il existe une multiplicité d'esthétiques de la vidéo vernaculaire. L'esthétique la plus courante se caractériserait par une caméra portée, une basse définition (quoique de moins en moins), la naturalité de la mise en scène, des moyens de productions parfois rudimentaires, l'improvisation et une faible post-production. Obéissant à des contraintes pragmatiques, celle-ci est depuis codifiée selon les systèmes de croyances propres à l'art et au cinéma contemporains - devenant une forme de plus en plus mobilisée, presque à la mode. Il y aurait une fétichisation de la forme vidéo vernaculaire qui devient une esthétique reconnaissable une fois qu'elle est adoptée et détournée parfois de manière critique par les artistes ou les cinéastes, qu'elle est arrachée à son espace d'origine et montrée dans d'autres espaces et contextes socioculturels. Ce style vernaculaire est désormais imité par des acteurs de la culture la plus hégémonique, telle clip musical de Beyoncé $7 / 11$, qui, simulant un tournage domestique réalisé à la Go-Pro dans une chambre d'hôtel, emprunte à la vidéo vernaculaire son imagerie, y ajoute une stratégie visuelle basée sur un effet d'authenticité et naturalité, une forme décontractée et apparemment sans prétentions artistiques ${ }^{15}$. Publié le 21 novembre 2014, le clip compte aujourd'hui plus de 500 millions

15. Le clip est disponible ici: https://www.youtube.com/watch?v=k4YRWT_Aldo. De nombreux articles de presse l'ont qualifié de "fait maison", "naturel”, "simple”. Par exemple, un article de L'Express publié lors de la sortie du clip, affirme: «Les images, simples également, sembleraient presque tournées à l'aide d'un smartphone par Jay-Z! » (https:// www.lexpress.fr/culture/musique/video-beyonce-se-la-joue-etonnamment-simple-dans-son-nouveau-clip-7-11 1624904. $\underline{\mathrm{html}}$ ); un autre texte, sur le media ChérieFM parlait d'une "parenthèse intimiste" (https://www.cheriefm.fr/artistes/ beyonce/actus/7-11-quand-beyonce-s-offrait-une-parenthese-intimiste-71421104). Un autre exemple plus récent est 
de vues sur YouTube. Ce clip, ainsi que d'autres exemples professionnels et commerciaux provenant des industries culturelles, permettent de valider les manières de faire de la vidéo vernaculaire auprès de ces créateurs, les vidéastes qui se trouvent notamment sur les plateformes d'hébergement vidéo.

Un dernier point reste à aborder. Il concerne la manière avec laquelle le cinéma peut potentiellement subvertir plutôt que réaffirmer l'hégémonie de la culture capitaliste, surtout quand les créateurs de ces images, ainsi que les cinéastes-troubadours, l'ont intégrée. Premièrement, ni YouTube, ni la plupart des réseaux sociaux numériques et des plateformes d'hébergement mainstream, entendus comme étant des instances de pouvoir dominantes sur le plan socio-économique, ne peuvent être pleinement considérés comme des bases de données, des (an)archives du vernaculaire audiovisuel ${ }^{16}$. YouTube n'est pas un espace neutre et démocratique, mais une entreprise capitaliste, appartenant à Google, qui obtient le contrôle, les bénéfices économiques, les droits et la propriété intellectuelle des contenus qui y sont publiés et hébergés. Plus encore, YouTube fonctionne sur le principe d'une gouvernementalité algorithmique qui met en place des hiérarchisations opaques des contenus modulant l'écologie de l'attention de ses utilisateurs. De ce fait, si le vernaculaire et le non-vernaculaire coexistent sur cette plateforme, les vidéos vernaculaires y sont les moins vues: le populaire, ce qui est fait par le peuple, fait partie des contenus les moins populaires, souvent marginalisés par l'intelligence artificielle de la plateforme. Ainsi, depuis quelques années, les plateformes d'hébergement vidéo sont en train de se professionnaliser et de s'institutionnaliser et le poids de la publicité diffusée y augmente (Kim). Il s'est produit une récente migration de la vidéo vernaculaire vers d'autres espaces, tels que TikTok, des sites pornographiques ou des plateformes de live streaming. Du reste, le paradoxe de la rentabilisation des contenus vernaculaires sur les plateformes - le cas extrême étant celui des YouTubeurs à grand succès (Holland) - confirme que si l'on adopte les critères économiques prônés par Illich (1983) pour délimiter ce qui relève du domaine vernaculaire, tous ces contenus dépendent des instances de pouvoir socio-économiques et n'ont aucune autonomie par rapport au marché capitaliste. Comment développer de véritables territoires de la vidéo vernaculaire reste une question ouverte, même si on peut d'ores et déjà affirmer que cela passerait par la création et la démocratisation de plateformes gérées selon les principes de la culture et du logiciel libres.

Bande-annonce de Roman National.

https://vimeo.com/264321378. BEIL, Grégoire, réal. Roman National. Bad Manners, 2018.

le clip du chanteur espagnol C Tangana «Nunca estoy», qui a cumulé en dix jours presque trois millions de vues sur YouTube.

16. À propos des tensions entre vernaculaire et institutionnel sur YouTube, voir, par exemple, Edgar (2013). 


\section{QEUVRes CITÉES}

\section{Ouvrages cités}

ALLARD, Laurence «Express Yourself 3.0! Le mobile comme media de la voix intérieure. Entre double agir communicationnel et continuum disjonctif soma-technologique». Téléphone mobile et création. Dir. Laurence Allard, Laurent Creton et Roger Odin. Paris : Armand Colin, 2014a. 139-61.

ALLARD, Laurence. «La vidéo conversationnelle: jouer avec les images, faire de sa vie un ready made by mobile (vine, snapchat, dubsmash, meerkat, periscope, riff, etc.)». Mobactu (décembre 2014b). URL: http://www.mobactu.org/la-video-conversationnelle-jouer-et-parler-avec-les-images-vine-snapchat-storydubsmasher/ (page consultée le 14 novembre 2020).

ALLARD, Laurence. «Partages créatifs: stylisation de soi et appspérimentation artistique». Communication \& langages 194.4 (2017): 29-39.

APPRICH, Clemens. «Remaking Media Practices: From Tactical Media to Post-Media». Provocative Alloys: A Post-Media Anthology. Dir. Clemens Apprich, Josephine Berry Slater, Anthony Iles et Oliver Lerone Schultz. Londres, Lüneburg: Post-media Lab \& Mute Books, 2013. 122-42.

AUFDERHEIDE, Patricia. «Vernacular Video: For the Growing Genre of Camcorder Journalism, Nothing Is Too Personal». Columbia Journalism Review 33.5 (janvier-février 1995) : 46-48.

BOËX, Cécile. «Surgissement, fragilité et effacement des vidéos vernaculaires de la révolution en Syrie». Communication lors de la journée d'étude Penser la révolution iranienne au temps présent. Paris, Le Bal (11 février 2019).

BOYD, danah. Taken Out of Context: American Teen Sociality in Networked Publics. Thèse de doctorat en Systèmes et Management de l'Information. Université de California, Berkeley, 2008.

BRATU HANSEN, Miriam. «The Mass Production of the Senses: Classical Cinema as Vernacular Modernism». Modernism/modernity 6.2 (avril 1999): 59-77.

BRUNS, Axel. Blogs, Wikipedia, Second Life and Beyond: From Production to Produsage. New York: Peter Lang, 2008.

BURGESS, Jean. Vernacular Creativity and New Media. Thèse de doctorat en Industries Créatives. Queensland University of Technology, 2007.

BURGESS, Jean et Joshua GREEN. YouTube. Online Video and Participatory Culture. Cambridge: Polity Press, 2009. 
CASILLI, Antonio. En attendant les robots. Paris: Seuil, 2019.

CHARTIER, Roger. «De l'écrit sur l'écran. Écriture électronique et ordre du discours». Communication lors du colloque Les écritures d'écran: histoire, pratiques et espaces sur le Web. Aix-en-Provence, Maison Méditerranéenne des Sciences de l'Homme (18 et 19 mai 2005). URL: https://imageson.hypotheses. org/658\#footnote 9658 (page consultée le 14 novembre 2020).

CHATONSKY, Grégory. «Post-Internet: époque, ontologie et stylistique». 2015. URL: http://chatonsky.net/ post-internet-definition/ (page consultée le 14 novembre 2020).

CHÉROUX, Clément. Vernaculaires. Essais d'histoire de la photographie. Paris: Le Point du Jour, 2013.

DEBRAY, Régis. Vie et mort de l'image. Une histoire du regard en Occident. Paris: Gallimard, 1992.

DERRIDA, Jacques. Mal d'archive. Une impression freudienne. Paris : Galilée, 1995.

EDGAR, Amanda Nell. «YouTube's "Bad Romance”: Exploring the Vernacular Rhetoric of Lady Gaga Parody Videos». The Journal of Social Media in Society 2.1 (printemps 2013) : 8-29.

FONTCUBERTA, Joan. La furia de las imágenes. Notas sobre la postfotografía. Barcelona: Galaxia Gutenberg, 2016.

GUNTHERT, André. «La visibilité des anonymes». Questions de communication 34 (2018). URL: https:// journals.openedition.org/questionsdecommunication/15693 (page consultée le 14 novembre 2020).

HAUSER, Gerard A. Vernacular Voices: The Rhetoric of Publics and Public Spheres. Columbia: University of South Carolina Press, 1999.

HERNÁNDEZ LÓPEZ, Gala. «Contes d'été virtuels. Le surcyclage de la vidéosphère dans Roman National ». L'art tout contre la machine. Dir. Rodolphe Olcèse et Vincent Deville. Paris : Hermann, 2021.

HETRICK, Judi. «Amateur Video Must Not Be Overlooked». The Moving Image 6.1 (janvier 2006) : 66-81.

HOGGART, Richard. La culture du pauvre. Étude sur le style de vie des classes populaires en Angleterre. 1957. Trad. de l'anglais par Françoise et Jean-Claude Garcias et Jean-Claude Passeron. Paris : Ed. de Minuit, 1991.

HOLLAND, Margaret. «How YouTube Developed Into a Successful Platform for User-Generated Content». Elon Journal of Undergraduate Research in Communications 7.1 (2016). URL: http://www.inquiriesjournal.

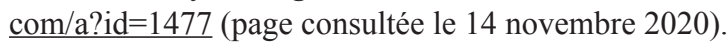

HOWARD, Robert Glenn. «The Vernacular Web of Participatory Media». Critical Studies in Media Communication 25.5 (décembre 2008) : 490-513.

ILLICH, Ivan. Le genre vernaculaire. Paris : Seuil, 1983.

ILLICH, Ivan. Le travail fantôme. Paris : Seuil, 1981. 
ILLICH, Ivan. Euvres complètes. Volume 2. Paris: Fayard, 2005.

JENKINS, Henry. Convergence Culture: Where Old and New Media Collide. New York: New York University Press, 2006.

JENKINS, Henry. «Vernacular Creativity: An Interview with Jean Burgess». (7 octobre 2007). URL: http:// henryjenkins.org/blog/2007/10/vernacular_creativity_an_inter.html (page consultée le 14 novembre 2020).

KIM, Jin. «The Institutionalization of YouTube: From User-Generated Content to Professionally Generated Content». Media Culture \& Society 34.1 (janvier 2012): 53-67.

LOVINK, Geert et Sabine NIEDERER. Video Vortex Reader. Amsterdam : Institute of Network Cultures, 2008.

LUNE RIBONI, Ulrike. “Juste un peu de video”. La vidéo partagée comme langage vernaculaire de la contestation: Tunisie 2008-2014. Thèse de doctorat en Sciences de l'Information et de la Communication, École Doctorale Sciences Sociales, Université Paris 8, 2016.

LUNE RIBONI, Ulrike. «Images anonymes, registres de visibilité et espace(s) public(s)». Questions de communication 35 (2019): 153-169.

MARROU, Henri-Irénée. Les Troubadours. Paris : Seuil, 1971.

McINTYRE, Lee. Post-truth. Cambridge: The MIT Press, 2018.

McLUHAN, Marshall. The Gutenberg Galaxy: The Making of Typographic Man. Londres: Routledge, 1962.

MEKAS, Jonas. Movie Journal: The Rise of the New American Cinema. 1959-1971. New York: Columbia University Press, 2016.

NEEMAN, Elsa, en collaboration avec Jérôme MEIZOZ et Claire CLIVAZ. «Culture numérique et auctorialité : réflexions sur un bouleversement». A contrario 17 (2012): 3-36.

ODIN, Roger. «La question de l'amateur ». Communications 68 (1999, «Le cinéma en amateur», dir. Roger Odin) : 47-89.

ONO, Kent A. et John M. SLOOP. «The Critique of Vernacular Discourse», Communication Monographs 62.1 (1995): 19-46.

RITZER, George et Nathan JURGENSON. «Production, Consumption, Prosumption: The Nature of Capitalism in the Age of the Digital "Prosumer"». Journal of Consumer Culture 10 (2010) : 13-36.

RODOVALHO, Beatriz. Amateur d'amateurs. Enjeux esthétiques et historiques du remontage de films de famille à travers l'œuvre de Péter Forgács. Thèse de doctorat en Études Cinématographiques et Audiovisuelles, École doctorale Arts et Médias, Université Sorbonne Nouvelle - Paris 3, 2018. 
ROUVROY, Antoinette et Thomas BERNS. «Gouvernementalité algorithmique et perspectives d'émancipation. Le disparate comme condition d'individuation par la relation?». Réseaux 177 (2013): 163 - 196.

SAPIO, Giuseppina. La pratique des home movies. Culture audiovisuelle et genèse de la méta-famille. Thèse de doctorat en Études Cinématographiques et Audiovisuelles, École doctorale Arts et Médias, Université Sorbonne Nouvelle - Paris 3, 2015.

SHERMAN, Tom. «Vernacular video». Video Vortex Reader: Responses to YouTube. Dir. Geert Lovink et Sabine Niederer. Amsterdam: Institute of Network Cultures, 2008. 161-168.

SNICKARS, Pelle et Patrick VONDERAU, dir. The YouTube Reader. Stockholm : National Library of Sweden, 2009.

SNOWDON, Peter. «The Revolution Will Be Uploaded: Vernacular Video and the Arab Spring». Culture Unbound, Journal of Current Cultural Research 6.2 (2014) : 401-429.

SPIVAK, Gayatri Chakravorty. «Can the Subaltern Speak? ». Marxism and the Interpretation of Culture. Dir. Lawrence Grossberg et Cary Nelson. Urbana: University of Illinois Press, 1988. 271-313.

SZENDY, Peter. Le supermarché du visible. Essai d'iconomie. Paris : Éditions de Minuit, 2017.

TOFFLER, Alvin. La Troisième Vague. Trad. de l'anglais par Michel Deutsch. Paris: Denoël, 1980.

ZABUNYAN, Dork. L'insistance des luttes. Lyon: De l'Incidence Éditeur, 2017.

\section{Cuvres filmiques citées}

BEIL, Grégoire, réal. Roman National. Bad Manners, 2018.

GAGNON, Dominic, réal. Going South. Dominic Gagnon, 2018.

ZHU, Shengze, réal. Present. Perfect. Burn the Film, 2019. 